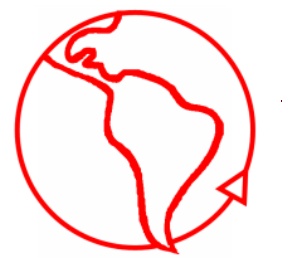

\title{
EDITORIAL
}

REVISTA MAD - UNIVERSIDAD DE CHILE, Nº 31 (2014)

\section{Hugo CADENAS}

Departamento de Antropología, Universidad de Chile.

Editor Revista Mad - Universidad de Chile.

El presente número de la Revista Mad-Universidad de Chile se puede catalogar como una unidad múltiple, como la una unidad de una diferencia. En esta edición aparecen un conjunto de investigaciones que abarcan temas tan diversos como la inclusión y exclusión, la percepción, la religión, la astronomía y los derechos humanos. El interés que motiva a la mayoría de estos trabajos es una problematización desde la teoría de sistemas sociales y los esfuerzos destinados a este tipo de explicación son, sin lugar a dudas, enormes y con resultados fructíferos.

La primera plana inicia con el artículo de Cornelia Bohn titulado "Inclusión y exclusión monetaria". En dicho texto se presenta un cuidadoso estudio acerca de las fronteras de la inclusión y la exclusión a través del medio de comunicación simbólicamente generalizado del dinero. Se propone problematizar el carácter inclusivo o exclusivo del dinero mediante una distinción adicional: centro, semiperiferia y periferia. El artículo explora las dimensiones de este problema.

A continuación Lionel Lewkow nos presenta su artículo "Aspectos sociológicos del concepto de percepción en la teoría de sistemas sociales", donde busca delinear un concepto de percepción basado en la teoría de sistemas sociales de Niklas Luhmann. A partir del análisis de la comunicación, se indagan los vínculos de los sistemas sociales con los sistemas psíquicos. El texto ejemplifica su postura a partir del sistema social de la ciencia y del medio de comunicación simbólicamente generalizado del amor.

Cierra esta sección el artículo de Marco Ornelas titulado "Lutero y el luteranismo: El buzón postal Martín Lutero". El artículo se concentra en el esquema persona/actor desde la teoría de sistemas sociales y ejemplifica 
dicha problemática a partir de la figura del reformador alemán Martín Lutero. A partir de este esquema se busca establecer una diferencia entre la persona de Martín Lutero y la doctrina que inspiró.

La sección aportes se inicia con el artículo de Alejandro Espinosa Rada titulado "Diferenciación de la astronomía: Autodescripciones y concepción heliocéntrica en el horizonte de la sociedad mundial". En este artículo se explora la génesis de una sociedad mundial a partir de la diferenciación de la astronomía. El artículo analiza un conjunto de modelos de explicación de la astronomía y su consecuencia para la diferenciación de la astronomía y la autodescripción de una sociedad en vías de mundialización.

Finalmente, cierra esta edición el artículo de Maximiliano Korstanje titulado "Duda y realidad: El uso político de los Derechos Humanos", donde se analiza de manera crítica el uso del problema de los Derechos Humanos por parte del actual gobierno argentino, aludiendo a una forma postmoderna de generar lo político, basada en una concepción de realidad particular.

Para finalizar esta editorial, quisiera agradecer a todos quienes participaron como evaluadores anónimos de los artículos recibidos. Gracias a su aporte voluntario y desinteresado, esta edición ha sido posible.

Revista Mad - Universidad de Chile

Envíos de artículos a: revistamad.uchile@gmail.com

Av. Capitán Ignacio Carrera Pinto 1045

CP 7800284, Nuñoa

Santiago de Chile

Chile

F: (+56 2) 29787760 